(GUD) and HSV-2. In the Ugandan and Kenyan trials, PCR did not find a sexually transmissible agent in $50-60 \%$ of GUD specimens, and MMC did not protect against HSV-2 in the Kenyan trial. We sought to better define the aetiology of GUD in the Kenyan trial and examine how MMC affects GUD aetiology.

Methods We defined GUD of unknown aetiology as negative for HSV type 1 and type 2, T pallidum, and $H$ ducreyi by PCR, and negative for HSV-2 and T pallidum by serology. In 59 GUD specimens from 59 men, 23 (39\%) had unknown aetiology. We identified bacterial microbiota in all 59 specimens using multitag pyrosequencing of the $16 \mathrm{~S}$ rRNA gene, and compared results by unknown vs STI aetiology. Statistical analysis employed exact methods.

Results Overall, 83 distinct genera were detected. Prevotella spp. was most abundant, accounting for $18 \%$ of microbiota on average, and present in $75 \%$ of specimens. Bacterial diversity was greater in GUD of unknown aetiology than STI associated GUD (median number of genera 13 [range 7-20] vs 11 [range 3-20], $\mathrm{p}=0.06$ ). Fusobacterium spp., Sneathia spp. and Anaerococcus spp. were more abundant in GUD of unknown aetiology $(7.7 \%, 7.6 \%$, and $7.3 \%$, respectively) than in GUD of STI aetiology (4.6\%, 5.0\%, and $5.4 \%$ ). Fusobacteriales (Fusobacterium spp. or Sneathia spp.) $[\mathrm{OR}=4.7 ; 95 \%$ CI: 1.3 to 19.9] and Anaerococcus spp. [OR=4.6; 95\% CI: 1.2 to 22.5] were more likely to be recovered in GUD of unknown aetiology than STI associated GUD. Fusobacteriales were more often recovered from uncircumcised men than circumcised men (62\% vs $22 \%$, $\mathrm{p}=0.04$ ), and Anaerococcus spp. was present in $22 \%$ of circumcised vs $70 \%$ of uncircumcised men $(p=0.010)$. Reported penile coital injuries were more common among men with Anaerococcus spp. $(85 \%$ vs $57 \%, p=0.01$ ), and condom use was less common ( $50 \%$ vs $71 \%$, $\mathrm{p}=0.11$ ). There was no difference in these bacteria by ulcer location. Conclusions Fusobacteriales and Anaerococcus spp. may colonise genital ulcers that develop from a mechanism related to circumcision status. Many such "ulcers" may be epithelial disruptions that are traumatic in origin. These bacteria have cytotoxic properties that may ulcerate or exacerbate pre-existing minor epithelial disruptions. MMC may reduce GUD through a reduction in these anaerobic bacteria.

\section{P3-S5.02 IS URETHRAL SMEAR MICROSCOPY IN ASYMPTOMATIC MEN EFFECTIVE IN REDUCING MAJOR M GENITALIUM INFECTION SEOUELAE IN WOMEN?}

doi:10.1136/sextrans-2011-050108.469

${ }^{5} \mathrm{P}$ White, ${ }^{1} \mathrm{R}$ Birger, ${ }^{2} \mathrm{~J}$ Saunders, ${ }^{2} \mathrm{C}$ Estcourt, ${ }^{1} \mathrm{~T}$ Hallett, ${ }^{3} \mathrm{O}$ Caffrey, ${ }^{4} \mathrm{C}$ Mercer, ${ }^{3,6} \mathrm{~T}$ Roberts. ${ }^{1}$ Imperial College, School of Public Health, London, UK; ${ }^{2}$ St Bartholomew's Hospital, London, UK; ${ }^{3}$ University of Birmingham, Birmingham, UK; ${ }^{4}$ University College London, London, UK; ${ }^{5}$ Health Protection Agency, London, UK; ${ }^{6}$ UK

Background Non-Chlamydial Non-Gonococcal Urethritis (NCNGU) is as prevalent as Chlamydial urethritis in UK men. Mycoplasma genitalium likely explains $10-46 \%$ of cases but there is no UKlicensed molecular test. In women, $M$ genitalium is associated with pelvic inflammatory disease (PID), infertility and ectopic pregnancy. New UK guidelines do not recommend urethral smear microscopy in asymptomatic men, so asymptomatic NCNGU cannot be diagnosed, whereas previously affected men and their sexual partners were treated with antibiotics. The impact of this policy on reproductive health sequelae in women is unknown.

Methods We used a compartmental mathematical model to synthesise evidence on the natural history of $M$ genitalium, diagnosis and treatment levels. Uncertainty was accounted-for using Latin hypercube sampling, with rejection sampling to fit to data. The model was stratified by sex, incorporates heterogeneous sexual behaviour, and was parameterised by behaviour data from key UK surveys (NATSAL2, PATSI, MSTIC); STI surveillance data; and natural history data from the literature. It incorporates symptomatic and asymptomatic infection; PID; care-seeking due to symptoms, partner notification, and routine screening; and treatment failure. We fitted to national STI surveillance (KC60) data, (allowing for uncertainty in the amount of NCNGU caused by $M$ genitalium), representing current practice, and examined (i) increasing microscopy in asymptomatic men by making it routine in all genito-urinary medicine clinics and (ii) decreasing rates by discontinuing it in the $20 \%$ of clinics currently still using it.

Results Microscopy in asymptomatic men reduces the burden of infection in women by reducing prevalence in men thus reducing transmission to women; and treating women via partner notification from diagnosed men. The magnitude of these effects depends upon natural history in men and women, about which there is uncertainty. We determine ranges of parameter values in which routine microscopy would be effective in reducing $M$ genitaliumrelated sequelae in women.

Conclusions Further empirical work is required to determine if natural history parameters fall in the ranges that would make routine microscopy in asymptomatic men effective. Particularly important are the prevalence of $M$ genitalium; proportion of infections that are symptomatic in men and women; duration of untreated infection; and incidence of PID, infertility and ectopic pregnancy attributable to $M$ genitalium.

\section{P3-S5.03 CONFLICT OF INTEREST AND POINT OF CARE TESTS: AN EXPLORATION OF ACCURACY IN HEPATITIS C INFECTION}

doi:10.1136/sextrans-2011-050108.470

${ }^{1} \mathrm{~S}$ Shivkumar, ${ }^{1} \mathrm{Y}$ Jafari, ${ }^{2} \mathrm{G}$ Lambert, ${ }^{2} \mathrm{C}$ Claessens, ${ }^{1} \mathrm{M}$ Klein, ${ }^{3} \mathrm{~J}$ Martinez-Cajas ${ }^{4} \mathrm{R}$ Peeling, ${ }^{1} \mathrm{~L}$ Joseph, ${ }^{1} \mathrm{~N}$ P Pai. ${ }^{1}$ McGill University, Montreal, Canada; ${ }^{2}$ INSPQ, Canada; ${ }^{3}$ Queen's University, Canada; ${ }^{4}$ LSHTM, UK

Background The WHO estimates that 170 million people worldwide are infected with Hepatitis C. In the context of HIV co-infection, rapid point-of-care tests gain importance in both the developing and developed countries. Moreover, in the light of the Food and Drug Administration's approval of the Oraquick point-of-care test for Hepatitis C for use in the USA, the accuracy of these tests is relevant.

Objective We conducted a systematic review and meta-analysis of the literature examining the sensitivity and specificity of all rapid point-of-care tests used to diagnose incident or prevalent Hepatitis $\mathrm{C}$, with an attention to involvement of industry in reporting of results.

Methods Two reviewers conducted independent searches of five databases between the years of 1995 and 2010. Bayesian metaanalysis was conducted accounting for the use of imperfect reference standards (sensitivity and specificity ranges of $90 \%-100 \%$ were assumed) in the assessment of index tests. The quality of all included full-text studies was assessed using the QUADAS and STARD checklists, with a focus on reporting of conflict of interest with industry.

Results A total of seven studies were identified from the database searches, of which five were conducted in developing settings. Eight index tests were examined including Oraquick, HCV Tri-Dot, HCV Bidot, Therma Ricerca, SM-HCV, Onecheck, Goldspot and Accurate. Sensitivity of all index tests ranged from $45 \%$ to $100 \%$, while specificity ranged from $93 \%$ to $100 \%$. Oraquick reportedly had the highest accuracy, with sensitivity ranging from $99 \%$ to $100 \%$ and a specificity of $100 \%$. However, the authors of the study reported a financial relationship with Orasure Technologies Inc., the makers of Oraquick. Although pooled sensitivity of all tests was high at 
$92.72 \%$ (95\% CI $72.11 \%$ to $99.93 \%$ ), when the Oraquick study was removed from analysis, the pooled sensitivity of all other tests dropped to $77.11 \%$ (95\% CI $45.49 \%$ to $99.61 \%$ ) see Abstract P3-S5.03 table 1. Pooled specificity remained high at almost 100\% regardless of whether the Oraquick study was included or not.

Conclusion Although Oraquick appears to be the most promising test, authors' ties with industry make these results less credible. More independent testing is required to be able to make policy recommendations for the most accurate index test to detect Hepatitis C infection.

Abstract P3-S5.03 Table 1 Results of bayesian meta-analysis: diagnostic accuracy of index tests used to detect Hepatitis C

\begin{tabular}{lll}
\hline Pooled results & Sensitivity (95\% CI) & Specificity (95\% CI) \\
\hline Including oraquick study & $92.72 \%(72.11 \%$ to $99.93 \%)$ & $99.88 \%(99.56 \%$ to $100 \%)$ \\
Excluding oraquick study & $77.11 \%(45.49 \%$ to $99.61 \%)$ & $99.99 \%(99.82 \%$ to $100 \%)$ \\
\hline
\end{tabular}

\section{P3-S5.04 THE DIAGNOSTIC ACCURACY OF RAPID POINT OF CARE TESTS USED TO DIAGNOSE HEPATITIS B: A BAYESIAN META-ANALYSIS}

doi:10.1136/sextrans-2011-050108.471

${ }^{1} \mathrm{~S}$ Shivkumar, ${ }^{1} \mathrm{Y}$ Jafari, ${ }^{2} \mathrm{G}$ Lambert, ${ }^{2} \mathrm{C}$ Claessens, ${ }^{1} \mathrm{M}$ Klein, ${ }^{3} \mathrm{~J}$ Martinez-Cajas, ${ }^{4} \mathrm{R}$ Peeling, ${ }^{1} \mathrm{~L}$ Joseph, ${ }^{1} \mathrm{~N}$ Pant Pai. ${ }^{1} \mathrm{Mc}$ Gill University, Montreal, Canada; ${ }^{2} \mathrm{INSPO}$, Canada; ${ }^{3}$ Queen's University, Canada; ${ }^{4}$ LSHTM, UK

Background More than 350 million people are infected with the Hepatitis B virus worldwide, with four million new cases every year. The prevalence of the Hepatitis B virus is highest in developing settings where laboratory equipment and diagnostic resources are limited. This creates a need for rapid point-of-care testing in order to screen blood donors and ensure timely diagnosis and treatment of infected individuals. Although studies have been conducted examining the accuracy of different tests, there has not been a synthesis of the available global evidence, or an assessment of the quality of evidence to date.

Objective We conducted a systematic review of the global literature examining the sensitivity and specificity of rapid point-of-care tests used to diagnose Hepatitis B, and meta-analysed the data. Additionally, we conducted a critical appraisal of the quality of included studies.

Methods Two reviewers conducted independent searches of five databases between the years of 1990 and 2010 for global evidence. Meta-analysis was performed grouping studies based on whether the index test identified $\mathrm{HBsAg}$, both $\mathrm{HBs}$ and eAg, or antibody to HbsAg. We used Bayesian meta-analysis, accounting for the fact that all of the studies used imperfect reference standards (sensitivity and specificity assumed to range between $90 \%$ and 100\%). The quality of all included full-text studies was assessed using the QUADAS and STARD checklists.
Results A total of 17 studies were identified, of which 13 were from developing countries and which investigated 22 different index tests. When studies were pooled, the Determine HBsAg test showed a combined sensitivity of $98.76 \%$ and a specificity of 99.94\%. Other HBsAg tests showed a lower combined sensitivity (96.77\%) but comparable specificity (99.89\%). The Amrad HBs + eAg test showed a combined sensitivity of $98.04 \%$ and a specificity of $99.04 \%$, while the tests detecting antibody to $\mathrm{HBsAg}$ showed a combined sensitivity of $99.77 \%$ and a specificity of $96.08 \%$. Studies were of poor-moderate quality with QUADAS scores ranging from 3 to 10/14 and STARD scores ranging from 7 to $14 / 25$ see Abstract P3-S5.04 table 1.

Conclusion The Amrad and Determine tests show the highest pooled accuracy. However, this could be explained by the fact that the other subgroups included studies examining different index tests with a wide range of accuracies. There is a need for more consistently designed studies, using ideal reference standards recommended by the CDC or Health Canada.

\section{P3-S5.05 RAPID POINT OF CARE TESTING FOR TEN SEXUALLY TRANSMITTED DISEASES}

doi:10.1136/sextrans-2011-050108.472

${ }^{1} \mathrm{~J}$ Kriesel, ${ }^{1} \mathrm{~A}$ Bhatia, ${ }^{2} \mathrm{M}$ Vaughn, ${ }^{2} \mathrm{~J}$ Gardner, ${ }^{3} \mathrm{C}$ Barrus, ${ }^{2} \mathrm{R}$ Crisp. ${ }^{1}$ University of Utah, School of Medicine, Salt Lake City, USA; ${ }^{2}$ ldaho Technologies Inc., Salt Lake City, USA; ${ }^{3}$ Salt Lake Valley Health Department, USA

Background Delays in the reporting of STD testing sometimes result in inappropriate patient care where a patient must be called back in for treatment. Some STDs may be missed because not all specimens are tested in a comprehensive manner. We are developing a multiplex point of care test to fill this clinical need. This fully automated system is capable of detecting ten PCR targets from a single specimen in $<1 \mathrm{~h}$

Methods A Sexually Transmitted Disease (STD) Panel was designed for the FilmArray device to detect the following organisms: Chlamydia trachomatis (CT), Neisseria gonorrhoeae (GC), Treponema pallidum, Trichomonas vaginalis, Mycoplasma genitalium, Ureaplasma urealyticum, Ureaplasma parvum, Haemophilus ducreyi, and herpes simplex viruses (HSV-1 and 2). Multiple PCR primers for each of these organisms were multiplexed and validated with the appropriate laboratory strains or plasmids.

Results Three hundred twenty-four subjects have been enrolled from the Salt Lake Valley Health Department STD clinic patient population, providing $600+$ specimens for analysis. Ninety-nine clinical specimens have been tested so far. The STD panel test results were compared to the standard CDC recommended clinical tests run in parallel on duplicate specimens. Standard testing included gram staining, CT/GC amplification, wet mount examination, viral culture, and serum syphilis IgG. Sample types included urine (44), vaginal/cervical swabs (7), urethral swabs (5), ulcer swabs (7), oral swabs (20), and rectal swabs (16). Concordance between the new STD panel and standard testing was: $C$ trachomatis (79/81, 98\%), N gonorrhoeae (81/81, 100\%), HSV1 (6/6, 100\%),

Abstract P3-S5.04 Table 1 Results from bayesian meta-analysis of diagnostic accuracy of hepatitis B point-of-care tests

\begin{tabular}{|c|c|c|c|c|}
\hline \multirow{2}{*}{$\begin{array}{l}\text { Program in R: Hierarchical } \\
\text { model } \\
\text { Subgroup }\end{array}$} & \multicolumn{2}{|c|}{ Assuming perfect reference standard $(100 \%)$} & \multicolumn{2}{|c|}{ Assuming imperfect reference standard $(90 \%-100 \%)$} \\
\hline & Sensitivity $(95 \% \mathrm{CI})$ & Specificity $(95 \% \mathrm{Cl})$ & Sensitivity (95\% Cl) & Specificity (95\% CI) \\
\hline $\mathrm{HBsAg}$ & $94.76 \%(90.08$ to $98.23 \%)$ & $99.54 \%(99.03$ to $99.953 \%)$ & $96.77 \%(92.92$ to $99.26 \%)$ & $99.89 \%(99.55$ to $100 \%)$ \\
\hline Determine-HBsAg & $98.24 \%(94.74$ to $99.98 \%)$ & $99.96 \%$ (99.31 to $100 \%)$ & $98.76 \%(94.67$ to $99.99 \%)$ & $99.94 \%(99.49$ to $100 \%)$ \\
\hline Amrad-HBs $+\mathrm{eAg}$ & $95.47 \%(88.88$ to $99.44 \%)$ & $99.81 \%(99.33$ to $100 \%)$ & $98.04 \%(93.39$ to $99.83 \%)$ & $99.95 \%(99.71$ to $100 \%)$ \\
\hline Anti-HBs & $93.15 \%(85.04$ to $98.5 \%)$ & $93.08 \%(81.9$ to $99.99 \%)$ & $99.77 \%(94.18$ to $100 \%)$ & $96.08 \%(86.38$ to $100 \%)$ \\
\hline
\end{tabular}

